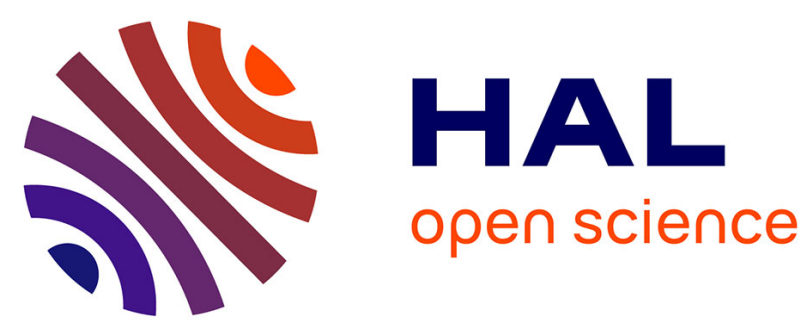

\title{
A SIMPLE INTERPRETATION OF ELECTRON LOCALISATION BEHAVIOUR IN WEAKLY DISORDERED SYSTEMS
}

\author{
C. Hodges
}

\section{> To cite this version:}

C. Hodges. A SIMPLE INTERPRETATION OF ELECTRON LOCALISATION BEHAVIOUR IN WEAKLY DISORDERED SYSTEMS. Journal de Physique Colloques, 1981, 42 (C4), pp.C4-67-C4-70. 10.1051/jphyscol:1981411 . jpa-00220726

\section{HAL Id: jpa-00220726 https://hal.science/jpa-00220726}

Submitted on 1 Jan 1981

HAL is a multi-disciplinary open access archive for the deposit and dissemination of scientific research documents, whether they are published or not. The documents may come from teaching and research institutions in France or abroad, or from public or private research centers.
L'archive ouverte pluridisciplinaire HAL, est destinée au dépôt et à la diffusion de documents scientifiques de niveau recherche, publiés ou non, émanant des établissements d'enseignement et de recherche français ou étrangers, des laboratoires publics ou privés. 


\title{
A SIMPLE INTERPRETATION OF ELECTRON LOCALISATION BEHAVIOUR IN WEAKLY DISORDERED SYSTENS
}

\author{
C.H. Hodges \\ Topexpress Limited, 1 Portugal Place, Cambridge, CB5 8AF, England
}

\begin{abstract}
A simple relation between the local energy spectrum and the time evolution of a wavepacket diffusing in a disordered system is discussed. In conjunction with polya's theorem for random walks, this relation implies an electron local density of states which in one and two dimensions is not square integrable over energy. such a local density of states is infinitely spiky however weak the disorder. It is suggested that this effect is a symptom of the localised electron eigenstates which always exigt in one and two dimensional disordered systems according to recent theories. By considering the smoothing of the local density of states as a function of inelastic scattering time one can deduce a time scale beyond which conventional weak-scattering transport theory breaks down. Assuming this breakdown is a manifestation of localisation one can obtain estimates of the localisation length. These agree with well established results for one dimension and the more recent scaling type of result for two dimensions.
\end{abstract}

It has been known for some time that the eigenstates of an electron moving in an infinite, one dimensional random potential are always localised in the Anderson sense. This remains true even when the potential fluctuations become very small, though, of course, the localisation length will then become very large. Recently a number of authors, for example [1], have proposed a qualitatively similar behaviour for localisation of eigenstates in a two dimensional random potential, arguing that a true Anderson transition from localised to extended states can only exist in three or more dimensions. These propasala have inspired a considerable amount of work, too voluminous to be cited here, most of which tends to confirm the non-existence of extended states in two dimensions. The majority of these investigations attempt to implement numerically the scaling or renormalisation group methods which are necessary if one wishes to understand the critical behaviour close to a mobility edge. However, there ought to be a simple interpretation for the absence of extended states in one and two dimensions which does not require these relatively sophisticated methods. In other manifestations of critical phenomena the frequent absence of transitions to ordered phases in lower dimensions was understood well before the renormalisation group appeared on the scene. such absences are in essence due to an infra-red divergence of the mean square fluctuation in order parameter which disrupts the ordered phase.

Recently I have proposed [2] a similax sort of interpretation for anomalous localisation behaviour in one and two dimensions. The idea is that diffusion theory and in particular polya's theorem for random walks implies fluctuations in the local density of states which 
always diverge in one and two dimensions. Polya's theorem states that any unrestricted random walk in one and two dimensions always returns eventually to the neighbourhood of its starting point while in three and higher dimensions there is a finite probability of escape [3]. Divergent fluctuations in the local density of states, are a necessary condition for a discrete local spectrum and provide strong circumstantial evidence for localisation. Moreover, if the states are assumed to be localised, the localisation length is found to agree with a well established result in one dimension [4] and the more recent scaling type of result in two dimensions [1]. In the present paper the argument will be reviewed critically.

My work is based on a relation between the local density of states $\rho(x, E)$ and the time dependence of wave intensity, which has also been discussed by other authors [5]. Consider the evolution from $t=-\infty$ to $t=+\infty$ of a wave packet

$$
\phi(r, t)=\sum_{n} f\left(E_{n}\right) \psi_{n}\left(r^{\prime}\right) \psi_{n}(r) \exp \left(-i E_{n} t / h\right)
$$

which is localised near $r=r^{\prime}$ at $t=0$. The eigenfunctions $\boldsymbol{\psi}_{n}$ are chosen to be real. The function $f(E)$ weights a particular region of enexgy but is broad enough to ensure localisation of a wavepacket at $t=0$ compared to any localipation length scale of the eigenfunctions themselves [2]. At the point of origin $r=r^{\prime}$ the time evolution of the wavepacket is related to the local density of states by

$$
\phi\left(r^{\prime}, t\right)=\int d E f(E) \exp (-i E t / h) \rho\left(x^{\prime}, E\right) .
$$

Because $\rho\left(x^{\prime}, E\right)$ is real the wave probability ia symmetric in time, $\left|\phi\left(r^{\prime}, t\right)\right|^{2}=\left|\phi\left(r^{*},-t\right)\right|^{2}$.

It is convenient to impose a time scale on the system by introducing an absorptive component to the potential which could correspond physically to inelastic scattering for example. We shall show that in one and two dimensions, fluctuations in the correspondingly blurred local density of states grow without limit as the inelastic scattering time $\tau_{i}$ tends to infinity. The fluctuating part of the local density of states is, by inverting equation (2),

$$
f(E) \tilde{\rho}_{\mu}\left(r^{\prime}, E\right)=h^{-1} f_{-\infty}^{+\infty} \exp (i E t / h) \exp (-\mu|t|) \tilde{\phi}\left(r^{\prime}, t\right) d t
$$

with $\mu=\tau_{i}^{-1}$ and $\tilde{\phi}$ the scattered part of the wavefield. This corresponds to convoluting $\tilde{\rho}_{0}\left(r^{\prime}, E\right)$ with a Lorenztian of width $\mu$.

Applying Parseval's theorem to equation (3) and taking the ensemble average, the mean square fluctuation is

$$
\left\langle\vec{\rho}_{\mu}\left(r^{\cdot}, E\right)^{2}\right\rangle=2 h^{-1}\langle\rho\rangle \int_{\tau}^{\infty} \mathrm{dt}\left\langle\left|\phi\left(r^{*}, t\right\rangle\right|^{2}\right\rangle \exp (-2 \mu|t|) .
$$

The lower limit to the integral in this equation is the mean free time for elastic backscattering. This is because we wish to exclude from equation (4) a small interval near $t=0$ over which the probability density is not effectively goverened by the diffusion equation. We also assume that for $t, \tau$ the unscattered wavepacket has left the origin $r^{\prime}$ so that we may set $\tilde{\phi}\left(r^{\prime}, t\right)=\phi\left(r^{\prime}, t\right)$. The probability density $|\phi|^{2}$ has been normalised to unity using equation (1). This introduces the average local density of states $\langle\rho \text {, and allows } f(E))^{2}$ to factor out [2]. For weak disorder $\langle\rho\rangle$ is close to the band structure 
value.

My interpretation of localisation behaviour in weakly disordered systems is based on the following supposition. If fluctuations $\tilde{\rho}_{\mu}$ are small relative to the mean $\langle\rho$ s then for times $t<\mu^{-1}$ the conventional semiclassical approach to diffusion theory is valid. Under these conditions such fluctuations have relatively little effect on the Fermi golden rule in the collision term of the Boltamann equation. The normalised probability density is then given by

$$
\left\langle|\phi(r, t)|^{2}\right\rangle=(4 \pi D t)^{-d / 2} \exp \left(-\left|r-r^{\prime}\right|^{2} / 4 D t\right)
$$

with d the dimensionality and the diffusion constant

$$
D=u^{2} T=u \lambda \text {, }
$$

$\lambda$ being the elastic mean free path for backscattering and $u$ the velocity. If on the other hand fluctuations become of the order of the mean on a critical time scale $T_{c}=\mu_{c}^{-1}$, then for $t>T_{c}$ the conventional semiclassical approach breaks down. At this stage a new constraint of a quantum mechanical nature enters the problem. This is because the density of states $\rho_{\mu}\left(r^{\prime}, E\right)$ is positive and therefore negative fluctuations $\tilde{\rho}_{\mu}\left(x^{\prime}, E\right)$ must be bounded in magnitude. It aeems reasonable that this new constraint should modify profoundiy the dynamics of the system.

It is not possible to prove that this breakdown is associated with Anderson localisation. But this is extremely plausible since if states are localised $T_{C}$ is precisely the time scale on which they begin to manifest themselves. Thus for such states $\mu_{C}$ is the separation between the delta functions of significant weight in the discrete local spectrum except in the unlikely event of clustering of these energy levels. The localisation length is then the diffusion radius [2]

$$
r_{l}=\left(D T_{C}\right)^{1 / 2}
$$

Values of $T_{c}$ and $r_{\ell}$ may be estimated by substituting equation (5) in equation (4) and applying the condition $\left.\left\langle\tilde{\rho}_{\mu}^{2}\right\rangle=\langle\rho\rangle\right\rangle^{2}$ for $\mu=\mu_{C}$. As $\mu \rightarrow 0$ the R.H.S. of equation (4) diverges 1 ike $\mu^{-0.5}$ in one dimension and $-\ln (\mu \tau)$ in two dimensions [6]. This divergence is equivalent to zero escape probability for random walks [3]. For $d \leqslant 2$ there is always a critical value for $\mu_{c}$ however weak the scattering, but not for $a>3$. One obtaing [2]

$$
\begin{gathered}
T_{\mathbf{C}} \sim \tau, \quad r_{\ell} \sim \lambda \quad(a=1) \\
T_{C} \sim \operatorname{Texp}(2 A k \lambda), r_{\ell} \sim \lambda \exp (A k \lambda) \quad(a=2)
\end{gathered}
$$

where $k$ is the de Broglie wavenumber um*/h and $A$ is of order unity. This is in agreement with previous results for one dimension [4] and the scaling type of result for two dimensions [1]. 
to exceed a critical strength before the conventional semiclassical approach breaks down over any time scale. Applying our criterion for breakdown, $\left\langle\tilde{\rho}_{\mu}^{2}\right\rangle$ of order $\langle\rho, 2$ with $\mu=0$, one deduces that localisation sets in when the elastic mean free path becomes of the order or less than the de Broglie wavelength $2 \pi / k$. This is just the Joffe Regel criterion for localisation.

In one and two dimensions there exist interpretations of equation (B) associated, like Joffe Regel criterion, with the uncertainty principle. In two dimensions ${ }_{c}$ can be shown to be the time scale for eventual return of random walks to within the de Broglie wavelength of their point of origin. This is also of the order of the time for interference between two independent random walks or wavepackets if the interference radius is the de Broglie wavelength.

We emphasize, once again that, above the percolation threahold, Anderson localisation is entirely a quantum mechanical effect. In our case all localisation lengths and time scales must tend to infinity as planck's constant $h$ tends to zero in the clasaical limit. At first sight the one dimensional results in equation (B) appeax to violate this condition, the interference time scale being independent of the interference radius. However, there is an implicit dependence of $T$ and $\lambda$ on Planck's constant, and in one dimension there is no backscattering of a classical particle above the percolation threshold.

The present work provides a simple interpretation and confirmation of much of the current thinking in Anderson localisation. However, the arguments above do not by themselves constitute a proof of the absence of extended states or quantum diffusion in two dimensions. Divergent fluctuations are a necessary, but not by themselves a sufficient condition for the local density of otates to be discrete. our work does nevertheless provide conclusive evidence that scaling arguments which predict a single "relevant" disorder to coupling parameter $w / v$ to scale to zero for weak disorder in two dimensions [7] cannot be correct. Such scaling behaviour must surely be associated with fluctuations in the local density of states, $i, e$. the wave intensities $\left|\psi_{n}\right|^{2}$, which are bounded.

\section{References}

[1] ABRAFAMS, E., ANDERSON, P,W, , LICCIARDELLO, D. C, and RAMAKRISHNAN, T.V., Phys, Rev. Lett. 42 (1979) 673.

[2] HODGES, C. H., J. Phys, C: SOl. St. Phys. 14 (1981) L247.

[3] ZIMAN, J. M., Models of Disorder (Cambridge : C.U.P.) 1979 p275.

[4] Thouless, D. J, , J. Phyg. C : Sol. st. Phyg. 6 (1973) L49.

[5] KIMball, J, C., J. Phys, C: sol, st. Phys. 11 (1978) 4347. Maldague, P. F., Phys, Rev. BL23 (1981) 1719.

[6] ABRAMOVITZ, M. and STEGUN, I,A., Handbook of Mathematical Functions (Dover : New York) 1965 p228.

[7] LEE, P. A., Phys. Rev. Lett. 42 (1979) 1492. 